\title{
On the Consistency of a Firm's Value with a Lognormal Diffusion Process
}

\author{
Andrew M. K. Cheung ${ }^{1}$, Van Son Lai ${ }^{2}$ \\ ${ }^{1}$ Department of Finance, Fairleigh Dickinson University, Vancouver, Canada \\ ${ }^{2}$ Department of Finance, Laval University, Quebec City, Canada \\ Email: amc111@fdu.edu, vanson.lai@fas.ulaval.ca
}

Received November 12, 2011; revised December 10, 2011; accepted December 30, 2011

\begin{abstract}
A partial equilibrium model is developed to examine conditions supporting the representation of the value of a firm by the lognormal diffusion process. The model formalizes the operating side of the firm and leads to a formula valuing the firm's risky profit stream. The present value formula is then compared to the existing work on valuing exogenous risky income stream. Implications of the resulted pricing model on the volatility of the firm value processes are explored.
\end{abstract}

Keywords: Cashflow Valuation; Adjustment Cost; Non-Constant Volatility Process; Lognormal Distribution

\section{Introduction}

Since the work of Merton [1] on pricing risky debt of a firm, it becomes a standard in the finance literature to assume a geometric Brownian motion representation of a firm's value process. Such a constant volatility lognormal distribution, the horsepower of option pricing, is rather consistent with some earlier influential papers by Rubinstein [2] and Ross [3]. These papers take the firm's risky investment cashflows as an exogenous stochastic process and then value these future income streams via an intertemporal arbitrary pricing operator.

In this paper, we explicitly model a firm that performs intertemporal profit maximization. Our model assumes there is a futures market for the firm's output. It specifies an internal production function for the firm and the adjustment cost function for its investment. This specification in conjunction with the external arbitrage market force leads to a present value formula for the firm's operating profit. Compared to one of the key results of Rubinstein [2], our main result unveils some severe restrictions behind the exogenous cashflow approach to a firm's value. Since the literature on the term structure of defaultable debt based on the constant volatility firm value process has not been empirically supported (see for instance Schonbucher [4]), our pricing formula also allows us to critically re-examine the firm value process. The main feature of our model imbeds a non-constant volatility value process while maintaining the tractable spirit of the classic structural approach to contingent claims analysis (CCA).

The rest of the paper is organized as follows. Section 2 describes the market setting. The firm's production acti- vity is introduced in Section 3. The present value of the firm's intertemporal profit and the resulting valuation equations are developed in Section 4 to 5. Section 6 concludes the paper.

\section{The Market Setting}

The analysis begins with a firm producing an output traded in a perfectly competitive market. The output price is assumed to follow an exogenous stochastic process

$$
\mathrm{d} P / P=\mu \mathrm{d} t+\sigma \mathrm{d} z
$$

where $\mathrm{d} z$ is the increment to a standard Brownian motion process, $\mu$ represents the expected growth rate of the output price and $\sigma$ stands for the instantaneous volatility of the output price. Both $\mu$ and $\sigma$ are assumed to be constant values rendering the conditional output price to be a lognormally distributed process.

Let $F(P, t)$ denote the futures price at time $t$ for delivery of one unit of the output at time $T$ and use $\tau=T-t$ to represent the remaining time to maturity. By Ito's le$\mathrm{mma}$, the instantaneous change in the futures price is given by

$$
\mathrm{d} F=\left(-F_{\tau}+1 / 2 F_{P P} \sigma^{2} P^{2}\right) \mathrm{d} t+F_{P} \mathrm{~d} P .
$$

The above equation represents the gains or losses generated by holding a futures contract. Uncertainty enters into a futures position through the second term. The risky component can be eliminated via a creation of the accompanying hedge portfolio. At time $t$ an investor can buy one unit of the commodity at a cost of $P(t)$ and simultaneously take a short position of $F_{P}^{-1}$ shares of 
futures contract. The futures position does not entail any initial cost.

The value of the hedge portfolio in the next instant is given by $\mathrm{d} P+\delta P \cdot \mathrm{d} t-F_{P}^{-1} \cdot \mathrm{d} F$ where the middle term rewards the owner of the commodity with the convenience of having the output on hand. In percentage terms, the return to the hedge portfolio is given by

$$
\begin{aligned}
& (\mathrm{d} P / P)+\delta \mathrm{d} t-\left(P F_{P}\right) \mathrm{d} F \\
& =\delta \mathrm{d} t-\left(P F_{P}\right)^{-1}\left(-F_{\tau}+1 / 2 F_{P P} \sigma^{2} P^{2}\right) \mathrm{d} t
\end{aligned}
$$

By virtue of the standard arbitrage argument forces the above deterministic portfolio return to be identical to the instantaneous return on the riskless asset $r \cdot \mathrm{d} t$. This implies the valuation partial differential equation (hereafter denoted as PDE) of the futures price is given by

$$
\delta \mathrm{d} t-\left(P F_{P}\right)^{-1}\left(-F_{\tau}+1 / 2 F_{P P} \sigma^{2} P^{2}\right) \mathrm{d} t=r \cdot \mathrm{d} t .
$$

Upon simplifying, we have

$$
1 / 2 F_{P P} \sigma^{2} P^{2}+(r-\delta) P \cdot F_{P}-F_{\tau}=0 .
$$

It can be readily verified that the solution to the PDE takes a simple form:

$$
F(P, t)=P(t) \cdot \exp [(r-\delta) \tau] .
$$

The following relation, a stochastic representation of the futures price process, is useful for the subsequent development of our main result:

$$
\mathrm{d} F=F_{P} \mathrm{~d} P-P \cdot F_{P}(r-\delta) \mathrm{d} t .
$$

\section{The Firm's Operating Profit}

The firm is assumed to operate in a perfectly competitive output market where there is no tax and the output fluctuates according to the geometric Brownian motion process. The firm's instantaneous revenue at time $s$ is generated by $P(s) Q(s)$ where $Q(s)$ is the firm's production function taking labor and capital as the input factors. We assume the firm's labor choice $L(s)$ can be made instantaneously whereas the adjustment cost assumption prohibits the firm to immediately obtain the desired capital stock.

Denote the investment variable as $I(t)$ and the capital stock $K(t)$; the relationship between these is defined by $\mathrm{d} K=\mathrm{I}(t) \mathrm{d} t$. The cost function associated with a given level of $I(t)$ is defined by $C(I)$. We assume that $C(I)$ is a convex cost function which is increasing in investment, such that $C^{\prime}(I)>0$ and $C^{\prime \prime}(I)>0$. Convexity of the cost function captures the reality that a high level of investment extracts limited resources from the firm to prepare for installation of additional capital stocks or to train labors with newly acquired machines. A convex adjustment cost function plays a key role in determining a finite size of the firm. Physical depreciation rate can be incorporated to the above stock and flow relation. However, for simplicity of exposition, we assume no depreciation.

Given the output of the firm at each instant, $Q(K, L)$, its net profit at time $t$ is defined by the difference between sales revenue and the relevant costs involved in producing the output:

$$
\pi(t)=P(t) Q(K, L)-w \cdot L(t)-C(I)
$$

While the management chooses the current level of labor combined with existing capital stocks to generate highest possible revenue, it has to devote resources to prepare for the future level of capital stocks in its production activity. The last term in the net profit equation for the firm then creates an intertemporal link between the current profit and the future profit for the firm, given the entire lifespan of the company, via the differential equation for the stock variable $K(t)$. Given the instantaneously adjustable choice variable $L$ and dynamic control variable $I$, the management takes the stochastic output price process $P(t)$ as the exogenous state variable. In this complete futures market, risk preference does not play a role in valuing the intertemporal profit of the business.

This implies there exists an equivalent martingale measure so that the firm evaluates its risky profit stream by using the risk free rate to discount the conditional expectation of its future net cash flow with respect to this martingale measure.

Maximization of the firm's net present value can be expressed as

$$
\begin{aligned}
& V(K(t), t)= \\
& \max _{I, L} E\left\{\exp (-r t) \int_{t}^{T} P(s) Q(L, K)-w \cdot L-C(I) \mathrm{d} s \mid \Phi_{t}\right\}
\end{aligned}
$$

where $\Phi_{t}$ represents the information set generated by the commodity price $P(t)$ and the expectation is taken with respect to the equivalent martingale measure. When the price process is specified as a lognormal diffusion, the information set can be substantially simplified. In this case $\Phi_{t}$ can be replaced by the currently observed value of the price process, $P(t)$.

\section{The Arbitrage Valuation of the Firm's Income Stream}

The net present value function indicates the business profit is derived from producing the homogeneous product that is sold at the market determined price $P$. At any future instant, the firm's profit is defined by combining the output price process with its production function. The valuation of these uncertain profit stream is parallel to that of determining the futures price in the earlier section. Denote the present value of the firm by $G(P, K, t)$. Consider the return to a hedge portfolio consisting of owning 
one unit of the firm's share and a short position on $G_{P} / F_{P}$ futures contracts. As the value of the firm is governed by the three state variables $K$ and $P$ and $t$, application of Ito's lemma leads to

$$
\mathrm{d} G=G_{P} \mathrm{~d} P+G_{K} \mathrm{~d} K+G_{t} \mathrm{~d} t+1 / 2 G_{P P}(\mathrm{~d} P)^{2},
$$

where the last term captures the Jensen's inequality representing the plausible non-linear relation between the firm's value and the output price. Recalling that the firm's current profit from producing the output is given by

$P \cdot Q-w \cdot L-C(I)$, we add these terms as income contributions to obtain the total change in the firm's value. On the other hand, the short futures position in the hedge portfolio generates the payoff given by

$\left(G_{P} / F_{P}\right) \mathrm{d} F$.

Combining the instantaneous value changes in each component of the hedge portfolio leads to

$$
\begin{aligned}
& G_{P} \mathrm{~d} P+G_{K} I \cdot \mathrm{d} t+1 / 2 G_{P P} \mathrm{~d} P^{2} \\
& +[P Q-w L-C(I)] \mathrm{d} t-\left(G_{P} / F_{P}\right) \mathrm{d} F \\
& =G_{K} I \cdot \mathrm{d} t+G_{t} \mathrm{~d} t+1 / 2 G_{P P} P^{2} \mathrm{~d} t+(r-\delta) P \cdot G_{P} \mathrm{~d} t \\
& \quad+[P Q-w L-C(I)] \mathrm{d} t .
\end{aligned}
$$

The right side of the above equation results from substituting the expressions for $\mathrm{d} P$ and $\mathrm{d} F$ from Equations (1) and (8) and simplifying. This equality indicates that the hedged portfolio return is non-stochastic. In the absence of arbitrage opportunity, the hedge portfolio return must grow at the riskfree rate leading to the following valuation $\mathrm{PDE}$ :

$$
\begin{aligned}
& 1 / 2 \sigma^{2} P^{2} G_{P P}+(r-\delta) \cdot G_{P}+G_{K} I \\
& +G_{t}+P Q-w L-C(I)=r \cdot G
\end{aligned}
$$

The solution function $G(P, K, t)$ to the partial differential equation represents the present value of the firm under a defined operating policy. The space of solution functions can be narrowed down and the solution form can be sharpened as soon as optimal choices to the control are made in the firm's decision problem and appropriate boundary conditions are specified.

\section{The Solution to the Profit Maximization Problem and the Value of the Firm}

This paper assumes a parametric form for the adjustment cost function $C(I)=\gamma I(t)^{\beta}, \gamma>0$ and $\beta>1$.

The parameter $\gamma \in(0, \infty)$ measures the significance of the adjustment cost. When $\gamma=0$, adjustment cost does not play any role in determining the firm's profit maximizing activities. Unless the production is under decreasing return to scale, the size of the firm in this case will end up being indeterminate. The other extreme,

$\gamma=\infty$, captures the firm's capacity constraint; any capital expansion is met with an infinite expense incurred by the firm's operation.

Letting $\gamma$ fall between the two extreme parametric values, the constant parameter $\beta$ can be interpreted as a measure of the speed of adjustment to the newly installed capital stocks. The case of a linear cost function where $\beta=1$, when combined with a constant return to scale production function leads to a firm's profit function that is linear in the capital stock. The implication of having a linear adjustment cost function is that the speedy capital formation indicates an unbounded acquisition of new capital to maximize the firm's profit. The resulting firm's size is again indeterminate. The convex adjustment cost, represented by $\beta>1$, can be justified as placing a bound to the firm's size. The chosen adjustment cost function is then combined with the firm's production technology.

The latter is assumed to be the Cobb-Douglas production function $Q(K, L)=L(t)^{\alpha} K(t)^{1-\alpha}$, where $\alpha$ is assumed to be a constant and $0<\alpha<1$.

The above specification of the investment cost function and the production technology reduce the generality of our model but it is motivated by the search for a closed form solution to the valuation problem. To further enhance the tractability of the problem, we assume that the firm is infinitely long-lived, removing the calendar time as one of the three state variables in the partial differential equation. The consequent valuation PDE derived from the last section is reduced to

$$
\begin{aligned}
r G=1 / 2 \sigma^{2} P^{2} \cdot G_{P P}+(r-\delta) P \cdot G_{P} \\
\quad+\max _{I, L}\left\{G_{K} I+P \cdot\left(L^{\alpha} K^{1-\alpha}\right)-w L-\gamma I^{\beta}\right\} .
\end{aligned}
$$

Performing the required maximization and substituting the resulting optimal choices yield the nonlinear PDE

$$
\begin{aligned}
r G= & 1 / 2 \sigma^{2} P^{2} G_{P P}+(r-\delta) P \cdot G_{P} \\
& +\gamma\left[\left((1 / \gamma \beta) \cdot G_{K}\right)^{1 /(\beta-1)}\right]^{\beta}+\xi P^{1 /(1-\alpha)} K
\end{aligned}
$$

where $\xi=(1-\alpha)(\alpha / w)^{1 /(1-\alpha)}$. The above valuation equation for the firm's value is expressed in terms of the state variables $P$ and $K$ given the parameters of the production and cost functions. Appendix A shows that the above valuation PDE has a solution given by

$$
G(K, P)=\theta K+\frac{(\beta-1) \cdot \gamma(\theta /(\beta \gamma))^{\beta /(\beta-1)}}{r-\mu(\beta /(\beta-1))-1 / 2 \sigma^{2}\left(\beta(1-\alpha+\alpha \beta) /(1-\alpha)^{2}(\beta-1)^{2}\right)}
$$


where

$$
\begin{aligned}
& \theta=\frac{\xi P^{(1 /(1-\alpha))}}{r-\mu(1 /(1-\alpha))-\left(\alpha \sigma^{2} / 2(1-\alpha)^{2}\right)} \\
& \text { and } \xi=(1-\alpha)(\alpha / w)^{\alpha /(1-\alpha)}
\end{aligned}
$$

Discussions on this equation are in order. There are three sets of variables forming the inputs to the formula. The first set consists of the production technology parameter $\alpha$ and the per unit labor cost $w$. The second set consists of the adjustment cost technology parameters $\gamma$ and $\beta$ measuring the significance and speed of adjustment. These two sets of parameters are assumed to be constant. The last set consists of state variables $K$ and $P$. The former is deterministic and the latter stochastic with coefficients $\mu$ and $\sigma$. Finally, the market required return on the spanned source of uncertainty $\mathrm{d} z$ is given by the riskless interest rate under the risk neutrality argument.

The exogenous commodity price $P$, which is the fundamental source of value to the firm's profit stream, affects the firm's present value through a composite variable $\theta$ defined above. Since the composite variable appears in the two separate terms of the value function in (13), it is useful to isolate the discussion of the influence of $\theta$ channeled through these two terms. The first term is the product of $\theta$ and $K$. Given that $K$ is the existing capital stock owned by the firm, $\theta \cdot K$ is naturally interpreted as the total value contribution to the firm by the exiting capital. Financial economists define $\theta$ as the marginal revenue product of the firm's capital.

It is worth pointing out that $\theta$ has a noticeable format reminiscent of the present value of a perpetual income stream under certainty. This perpetuity interpretation is consistent with the presumption that the business is infinitely lived with its future risky stream of profit discounted by a complete arbitrage free financial market. It is now useful to compare $\theta$ in this paper with the earlier result derived by Rubinstein [2]. Rubinstein's model sets the standard methodology for firm's valuation problem in finance. Given an exogenous stochastic cashflow process for a business firm, an appeal to an efficient financial market governed by a martingale pricing operator is necessary and sufficient to produce a fair market value of the firm's cashflow. Rubinstein assumes a discrete stationary random walk process, which is a discrete counterpart of the geometric Brownian motion process for our commodity price process with a zero drift.

Our perpetuity reasoning for $\theta$ in this paper is different but consistent with Rubinstein's result.

The difference arises from the fact that the firm's production activity is endogenized and the technology parameter $\alpha$ plays a role in producing the transformed ex- pected growth of the commodity price via

$(1 /(1-\alpha)) \mu-(1 / 2)\left(\alpha /(1-\alpha)^{2}\right) \sigma^{2}$. The difference between the required market return $r$ and the expected growth opportunity stands for the market net required return used to discount the marginal revenue contribution by the installed capital.

The consistency of the first term with Rubinstein's result also allows us to emphasize the contribution of the second value component. The second term highlights the presence of the adjustment cost parameters $\gamma$ and $\beta$ that, when combined with the production parameter, further transform the expected growth of the commodity price process. As it takes time and resource for the firm to turn the raw capital into its ultimate production form, the firm has earned an access to the future benefit accrued by these new capital via the firm specific cost technology. Such adjustment cost associated benefit is spread over the indefinite future and the financial market discounts those benefits stream through an appropriately adjusted cost of capital. The result is the rational appearance of the second value component.

Two special cases arise from limiting arguments that would vanish the second term and reduce the present value formula to the standard result where value arises mainly from the firm's production technology. The first case corresponds to no adjustment cost incurred when new capital is acquired $(\gamma=0)$. The second case arises when the adjustment cost function is linear in investment $(\beta=1)$. Substituting either one of the these cases is sufficient to reduce the second term of the value function to zero. As discussed earlier, both cases correspond to a situation where the firm's size is indeterminate and the intertemporal optimization problem has no interior solution. The standard perpetuity formula in the finance literature appears to thrive on the validity of these two cases.

An additional disquieting feature of the valuation formula begins to surface when one continues examining the stochastic evolution of the valuation function $G(K, P)$. Whereas the commodity price process follows a simple geometric Brownian motion with a constant volatility, the resulting process for $G$ is not a geometric Brownian motion with a constant volatility. A causal observation ${ }^{1}$ of the functional form for $G$ suggests this consequent feature. Some lengthy algebraic developments are presented in the Appendix B to verify this claim.

In that appendix it is also shown that either $\gamma=0$ or $\beta=1$ would allow one to restore the geometric Brownian motion representation for $G$. On the contrary, when the firm possesses a significant convex adjustment cost

\footnotetext{
${ }^{1}$ The fact that $\beta$ and $\gamma$ appear more than once in the second term of the pricing formula does not simplify that term immediately to zero when evaluated at $\beta=1$ or $\gamma=0$. Some delicate limiting arguments are established in Appendix B to justify that this second term reduces to zero.
} 
function, one does not have a lognormal diffusion representation for its value process. The implication of this analysis has some nontrivial bearing on many existing models that rely on assuming a value process for a firm's assets following a geometric Brownian motion with a constant volatility. Although the popular lognormal diffusion model gives rise to numerous useful mathematical features and valuable economic insights in finance, our analysis has uncovered the severe limitations imposed on the business entity when the constant volatility assumption is adopted.

\section{Conclusions}

This paper begins with a neoclassic firm model and explores conditions leading to the lognormal diffusion price process that becomes the standard exogenous stochastic process in modeling a firm's value process since the work of Merton [1]. There are works in finance literature that traces the economic connection between the lognormal diffusion process and the general equilibrium fundamentals. Such interesting connection is essentially behind the term viable price process after Bick's [5] influential analysis. The result of this paper is based on a partial equilibrium firm value model in a complete market setup which keeps the representative agent behind the risk neutral probability. In the end, the geometric Brownian motion value process with a constant volatility emerges as a special case of a more general adjustment cost technology. The resulting non-geometric Brownian motion value process can also be qualified as a viable firm value process.

In standard option pricing models, the assumption that stock prices follow a geometric Brownian motion processes has long been criticized as lacking empirical supports. Proponents of the non-constant volatility model emphasize the need to add random volatility and jumps in the generalization of the original Black-Scholes model. The notion that volatility is a non-diversifiable exogenous process turns the original Black-Scholes option pricing environment into an extended two state variables pricing framework.

Earlier works of Hull and White [6], Scott [7] and Heston [8], while offering substantial insights to the extended pricing framework, add necessary economic and computational complications. This paper is aligned with the extended constant volatility literature, but it aims at producing a tractable result on a firm's value with only one state variable. The next task is to take the implication of the present paper to modify some of the existing works that are crucially based on a geometric Brownian motion process for the firm asset values, the horsepower of Merton [1] seminal structural approach to corporate securities.

\section{Acknowledgements}

The comments from Matthew Morin on an earlier draft of this paper and the formatting assistance from Arsia Assadipour are greatly appreciated. Lai acknowledges financial support from the Fonds Conrad Leblanc and the Social Sciences and Humanities Research Council of Canada (SSH $\mathrm{RC}$ ).

\section{REFERENCES}

[1] R. C. Merton, "On the Pricing of Corporate Debt: The Risk Structure of Interest Rates," Journal of Finance, Vol. 29, No. 2, 1974, pp. 449-470. doi:10.2307/2978814

[2] M. Rubinstein, "The Valuation of Uncertain Income Streams and the Pricing of Options," Bell Journal of Economics and Management Science, Vol. 7, 1976, pp. 407-425.

[3] S. A. Ross, "A Simple Approach to the Valuation of Risky Streams,” Journal of Business, Vol. 51, No. 3, 1978, pp. 453-475. doi:10.1086/296008

[4] P. J. Schonbucher, "Credit Derivatives Pricing Models," John Wiley and Sons, New York, 2003.

[5] A. Bick, "On the Consistency of Black Scholes Model with a General Equilibrium Framework," Journal of Financial and Quantitative Analysis, Vol. 22, No. 3, 1987, pp. 259275. doi: $10.2307 / 2330962$

[6] J. C. Hull and A. White, "The Pricing of Options on Assets with Stochastic Volatilities," Journal of Finance, Vol. 42, No. 2, 1987, pp. 218-300. doi:10.2307/2328253

[7] L. O. Scott, "Option Prices When the Variance Changes Randomly," Journal of Financial and Quantitative Analysis, Vol. 22, No. 4, 1987, pp. 419-438. doi: $10.2307 / 2330793$

[8] S. Heston, "A Closed Form Solution for Options with Stochastic Volatility with Applications to Bond and $\mathrm{Cu}-$ rrency Options," Review of Financial Studies, Vol. 6, No. 2, 1993, pp. 327-345. doi:10.1093/rfs/6.2.327

[9] R. Goldberg, "Methods of Real Analysis," John Wiley and Sons, New York, 1976. 


\section{Appendix A}

In this appendix we derive the solution to the non-linear PDE stated as Equation (12) in Section 5. We conjecture the following solution function

$$
G(K, P)=A(P) K+B(P)
$$

where $A(P)$ and $B(P)$ are functions assumed to be at least twice continuously differentiable with respect to the variable $P$. Then the valuation PDE take an additively separable form

$$
\begin{aligned}
& r\{A(P) K+B(P)\}=1 / 2 A_{P P} K \sigma^{2} P^{2} \\
& +A_{P} K(r-\delta) P+\xi P^{1 /(1-\alpha)} K+1 / 2 B_{P P} \sigma^{2} P^{2} \\
& +B_{P}(r-\delta) P+\gamma(\beta-1)\left[((1 /(\gamma \beta)) A)^{1 /(\beta-1)}\right]^{\beta} .
\end{aligned}
$$

First adopt a functional form $A(P)=q \xi P^{1 /(1-\alpha)}$ and we need to verify it satisfies the first segment of the entire PDE. It is a matter of taking the necessary partial derivatives, substituting the $A(P)$ and its derivatives on both sides of the above PDE. Then the unknown co-efficient q comes out to be

$$
q=\frac{1}{r-(1 /(1-\alpha))(r-\delta)-1 / 2(1 /(1-\alpha))^{2} \alpha \sigma^{2}} .
$$

Finally, substitute $q$ back into the conjectured solution gives

$$
\begin{aligned}
& A(P) \\
& =\frac{1}{r-(1 /(1-\alpha))(r-\delta)-1 / 2(1 /(1-\alpha))^{2} \alpha \sigma^{2}} \xi P^{1 /(1-\alpha)}
\end{aligned}
$$

We have half of the solution for $\mathrm{G}(\mathrm{P}, \mathrm{K})$ worked out as

$$
G(P, K)=A(P) K+B(P)=\theta K+B(P)
$$

where $\theta$ is the chosen notation in Section 5 and it is identical to $A(P)$. It remains to solve for $B(P)$. Let us conjecture $B(P)$ with the following solution form

$$
\begin{aligned}
B(P) & =b \gamma(\beta-1)\left((1 / \gamma \beta) q \xi P^{1 /(1-\alpha)}\right)^{\beta /(\beta-1)} \\
& =b H P^{\beta /(1-\alpha)(\beta-1)}
\end{aligned}
$$

where we set

$$
H=\gamma(\beta-1)((1 / \gamma \beta) q \xi)^{\beta /(\beta-1)}
$$

Next, substitute $B(P)$ into the remaining segment of the PDE with the corresponding partial derivatives appropriately taken in order to solve for the unknown coefficient $b$. The resulted $\mathrm{b}$ comes out to be

$$
b=\frac{1}{r-(r-\delta)(\beta /(1-\alpha)(\beta-1))-1 / 2 \sigma^{2}\left(\beta(1-\alpha+\alpha \beta) /(1-\alpha)^{2}(\beta-1)^{2}\right)}
$$

Further, putting $b$ back into the conjectured solution $B(P)$ gives

$$
B(P)=\left\{\frac{(\beta-1) \gamma(\theta / \beta \gamma)^{\beta /(\beta-1)}}{r-(r-\delta)(\beta /(1-\alpha)(\beta-1))-1 / 2 \sigma^{2}\left(\beta(1-\alpha+\alpha \beta) /(1-\alpha)^{2}(\beta-1)^{2}\right)}\right\} .
$$

Combining the verified solution forms for $A(P)$ and $B(P)$ gives

$$
G(K, P)=A(P) K+B(P)=\theta K+\frac{(\beta-1) \gamma(\theta / \beta \gamma)^{\beta /(\beta-1)}}{r-(r-\delta)(\beta /(1-\alpha)(\beta-1))-1 / 2 \sigma^{2}\left(\beta(1-\alpha+\alpha \beta) /(1-\alpha)^{2}(\beta-1)^{2}\right)}
$$

This completes the derivation of the claimed solution.

\section{Appendix B}

In this appendix, we examine the stochastic dynamics of the firm's value process given the closed form solution in Section 5. For convenience we recall Equation (12), the pricing formula,

$$
G(K, P)=\theta K+\frac{(\beta-1) \gamma(\theta / \beta \gamma)^{\beta /(\beta-1)}}{r-\mu(\beta /(1-\alpha)(\beta-1))-1 / 2 \sigma^{2}\left(\beta(1-\alpha+\alpha \beta) /(1-\alpha)^{2}(\beta-1)^{2}\right)}
$$

where

$$
\theta=\frac{\xi P^{(1 /(1-\alpha))}}{r-\mu(1 /(1-\alpha))-\left(\alpha \sigma^{2} / 2(1-\alpha)^{2}\right)}, \quad \xi=(1-\alpha)(\alpha / w)^{\alpha /(1-\alpha)}
$$


Since $P$ is the only stochastic state variable, let

$$
\begin{gathered}
D_{1}=\frac{\xi}{r-\mu(1 /(1-\alpha))-\left(\alpha \sigma^{2} / 2(1-\alpha)^{2}\right)} \\
D_{2}=\frac{(\beta-1) \cdot \gamma\left(D_{1} /(\beta \gamma)\right)^{\beta /(\beta-1)}}{r-\mu(\beta /(\beta-1))-1 / 2 \sigma^{2}\left(\beta(1-\alpha+\alpha \beta) /(1-\alpha)^{2}(\beta-1)^{2}\right)}
\end{gathered}
$$

Then $G(K, P)$ can be rewritten as

$$
G(K, P)=D_{1} P^{1 /(1-\alpha)} K+D_{2} P^{\beta /(1-\alpha)(\beta-1)} .
$$

Also, recall the commodity price dynamics is given as $(\mathrm{d} P / P)=\mu \mathrm{d} t+\sigma \mathrm{d} z$ and the capital stock dynamics is $\mathrm{d} K=I \cdot \mathrm{d} t$. Next, apply Ito's lemma to the function $G$ to obtain $\mathrm{d} G=\mu_{G} \mathrm{~d} t+(\partial G / \partial P) \sigma P \cdot \mathrm{d} z$. Our goal is to investigate whether the instantaneous return on the firm's value process will have a constant volatility, given that the volatility $\sigma$ to the commodity price process is a constant. To pursue this goal, it suffices to examine the stochastic part of the above $\mathrm{d} G$ process. Taking the partial derivative of the value function and rearranging, we obtain

$$
\begin{aligned}
\sigma P \frac{\partial G / \partial P}{G}= & {\left[\frac{\sigma D_{1}(1 /(1-\alpha))}{\left.1+D_{2} P^{1 /(1-\alpha)(\beta-1)}\right]}\right.} \\
& +\left[\frac{\sigma D_{2}(\beta /(1-\alpha)(\beta-1))}{D_{1} K \cdot P^{(-1 /(1-\alpha)(\beta-1))}+D_{2}}\right]
\end{aligned}
$$

where $P$ is non-vanishing in each of the two terms on the right hand side. We also want to examine the case when $\beta=1$. At this juncture we set aside some delicate issues involving the limiting value of the second term when $\beta$ approaches one. On the premise that the second term approaches zero when $\beta$ approaches one, we consider the simplified value function for the firm

$$
G(K, P)=D_{1} P^{1 /(1-\alpha)} K .
$$

In this case, taking the partial derivative and re-arranging we obtain

$$
\sigma P \frac{\partial G / \partial P}{G}=\sigma \cdot(1 /(1-\alpha)) .
$$

This verifies that when $\beta$ approaches one, the firm's instantaneous return process has a constant volatility.

We are left to examine the limiting value of the second term in the valuation equation when $\beta$ approaches one. When $\beta \rightarrow 1$, the value of the numerator tends to infinity because of the presence of $(\beta /(\beta-1))$ in the ex- ponent to $\theta / \beta \gamma, \theta, \gamma>0$. On the other hand $1 /(\beta-1)$ and $1 /(\beta-1)^{2}$ enter into the denominator in such a way to lead the entire denominator to approach negative infinity when $\beta \rightarrow 1$ The consequent ratio leads to an indeterminacy. Nevertheless, the following lemma, an adaptation of the generalized mean-value theorem, resolves the ambiguity. Before stating the lemma, let

$$
\begin{aligned}
& x=\beta-1, \Omega=\theta / \beta \gamma, g=\mu(\beta /(1-\alpha)), \\
& h=1 / 2 \sigma^{2}\left(\beta(1-\alpha+\alpha \beta) /(1-\alpha)^{2}\right)
\end{aligned}
$$

Next, we write the second term as

$$
W(x)=\frac{x \gamma \cdot \Omega^{\beta / x}}{r-g x^{-1}-h^{-2}} \equiv \frac{M(x)}{N(x)} .
$$

Lemma: Suppose $M(x)$ and $N(x)$ are differentiable functions, except at $x_{0}$, in $a<x<b$ and

$$
\lim _{x \rightarrow x_{0}} M(x)=\infty, \quad \lim _{x \rightarrow x_{0}} N(x)=\infty
$$

where $a<x_{0}<b$. Then

$$
\lim _{x \rightarrow x_{0}} \frac{M(x)}{N(x)}=\lim _{x \rightarrow x_{0}} \frac{M^{\prime}(\zeta)}{N^{\prime}(\zeta)}
$$

for $a<x_{0}<x<\zeta(x)<b$.

The proof of this result is found in Goldberg [9], p. 204. The intuition of the above lemma is that one can avoid the indeterminacy from the ratio of two infinities. Let us specialize the lemma to our second term in the $G(P, K)$ function and observe that letting $x_{0} \rightarrow 0$ is equivalent to setting $\beta \rightarrow 1$. Then

$$
\frac{M^{\prime}(\zeta)}{N^{\prime}(\zeta)}=\frac{\beta \gamma \ln \Omega \cdot \exp \left(\beta \zeta^{-1} \ln \Omega\right)}{r-2 g \zeta^{-1}-3 h \zeta^{-2}} .
$$

Provided $0<\Omega<1$, we have $\ln \Omega<0$. It follows that the above ratio pointwise converges to zero as $x \rightarrow x_{0}$. A similar argument can be developed to show that when $\gamma \rightarrow 0$ the second term of the valuation equation converges to zero. 